\title{
Mind-changing IBS therapy
}

A recent study has shown that hypnotherapy and educational intervention normalize the abnormal processing and enhanced perception of visceral stimuli in patients with IBS.

Previous studies have demonstrated the efficacy of hypnotherapy for the treatment of IBS, but Susanna Walter and colleagues wanted to investigate the effect of hypnotherapy and other mind-based interventions on brain responses to cued rectal distensions in patients with IBS.

44 right-handed women with moderate-to-severe IBS were included in the study, along with 20 healthy individuals as controls. Functional MRI (fMRI) was used to measure blood-oxygen-leveldependent (BOLD) signals in the brain during expectation and delivery of rectal distensions.

"Increased BOLD response in localized brain regions is interpreted as increased neural activity," explains Walter.

Patients who responded to hypnotherapy treatment (that is, who experienced symptom reduction) demonstrated a reduction in BOLD activity in both anterior insula and posterior insula during high intensity rectal distension. After treatment, brain responses were similar to those of healthy controls, indicating that these psychological interventions might have a normalizing effect on the central processing of visceral signals. Patients who responded to educational intervention also demonstrated attenuation in BOLD activity, but in different areas of the brain.

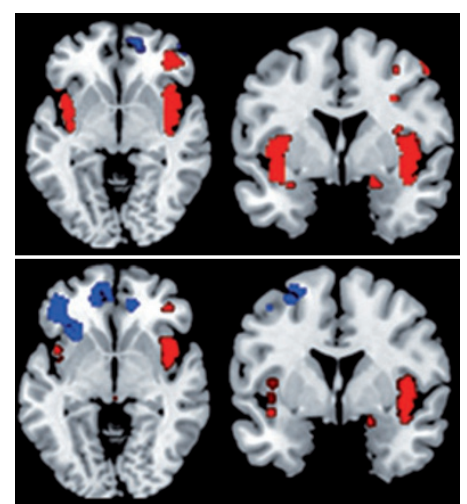

$B O L D$ response during rectal distension in patients with IBS. Top panel: Before hypnotherapy treatment. Bottom panel: After hypnotherapy treatment. Red represents increased BOLD response and blue represents decreased BOLD response. Abbreviation: BOLD, blood lood

"Hopefully studies such as this one will help to change minds by convincing people that response to such treatments results in clearly demonstrable changes within the brain rather than having to just rely on the subjective reporting of symptoms," says Peter Whorwell who is interested in the effects of hypnotherapy on IBS, but was not involved in the study.

Walter and co-workers conclude that, "Longitudinal studies are needed to determine the stability of the hypnotherapy-induced changes over time, as well as to determine if persistent symptomatic improvements are associated with normalization of structural brain changes (grey and white matter) recently identified in such patients."

Isobel Leake

Original article Lowén, M. B. O. et al. Effect of hypnotherapy and educational intervention on brain response to visceral stimulus in the irritable bowel syndrome. Aliment. Pharmacol. Ther. doi:10.1111/apt.12319 